\title{
Tackling Disability of Speech due to Stroke: Perspectives from Stroke Caregivers of the University Teaching Hospital in Zambia
}

\author{
${ }^{1 *}$ Brian Chanda Chiluba, ${ }^{2}$ Joseph Phiri \\ ${ }^{1,2}$ School of Health Sciences, University of Zambia, Lusaka, Zambia \\ ${ }^{3}$ Department of Physiotherapy-School of Health Sciences, University of Zambia, Lusaka, Zambia
}

\begin{abstract}
Many stroke survivors usually have permanent disability such as aphasia, which result in the need for support from caregivers. Care required for stroke survivors differs depending on the type of stroke, but little research has been done in this regard. The literature that exist provides unsatisfactory evidence for support services and interventions for caregivers, especially in relation to managing affective symptoms. The aim of this study was to explore the experiences of caregivers handling aphasic stroke patients at the University Teaching Hospital. An explorative study utilizing qualitative phenomenological approach was utilized with sample of 15 participants using a purposeful sampling technique. A thematic method of analysis was carried out. This study found that caregivers go through a lot of things in caring for their aphasic stroke patients and their experience involve a complicated and sometimes unpredictable interplay of multiple processes. Lack of preparedness in assuming the role of caregiving caused the caregivers to go through what seemed to be insurmountable challenges. There is therefore need for caregiver education for delivery of appropriate care for stroke survivors.
\end{abstract}

Keywords: Aphasia, Caregiver, Experiences, Stroke, Disability

\section{Research Background}

Stroke, the most common form of cerebrovascular disease, is a disabling medical condition that is particularly common in elderly populations with over 300,000 individuals left with moderate to severe disabilities such as aphasia [1]. Following discharge from the hospital, stroke survivors can be left with significant cognitive and physical impairments and may require long-term care and support [2]. This support is commonly provided by spouses, children or other relations, who become informal caregivers offering full-time care [3]. Therefore, stroke not only impacts upon the individual but also on their immediate and extended families. The typically sudden onset of stroke means that families can suddenly find themselves in a caring role. Despite their important role, caregivers are at an increased risk of experiencing negative biopsychosocial consequences and reduced quality of life [4].

\footnotetext{
* Corresponding author: Brian Chanda Chiluba

brian.chiluba@unza.zm

Published online at http://IJDS.ub.ac.id/

Copyright @ 2019PSLD UB Publishing. All Rights Reserved
}

While there has been considerable research into the pervasive effects of stroke on survivors [1], relatively little attention has been paid to the experiences of the primary caregiver. More specifically, the psychosocial changes these caregivers experience and their individual needs are still poorly understood [2]. The literature that is available on caregivers of aphasic stroke survivors provides evidence that caregiving is often challenging, affecting the physical and mental health of the caregiver [3]. Caregivers also experience significant dissatisfaction with available support services because their experiences and needs are often overlooked [2]. Unsurprisingly then, current research provides evidence that effective interventions for caregivers, especially in relation to managing emotional symptoms, remain inadequate [5].

Caregivers end up learning to cope with a multitude of new roles and responsibilities abruptly in order to help the aphasic stroke survivors [6]. Over time, if the burden of care is excessive, the caregiver's health may breakdown, with a subsequent loss of support for the 'patient' [7]. While there is extensive 
literature on caregiving [4], less is known about stroke caregivers' experiences over time and the skills and knowledge they require to facilitate caregiving.

While some researchers have tried to measure the prevalence of and risk factors for caregiver burden following stroke, many of them report inconsistent findings and fail to comprehensively explain caregiver experiences. It is still uncertain what factors underlie a caregiver's psychosocial and physical health and which caregivers cope better than others, and why this is the case. The reasons behind satisfaction and dissatisfaction with support services are also poorly understood [8].

\section{Method}

\subsection{Design and setting}

An explorative study utilizing qualitative phenomenological approach was employed to carry out this study. This was chosen because phenomenological studies investigate different people's perceptions of their experiences of a particular phenomenon via in-depth semi or unstructured interviews with a purposefully selected group of individuals, i.e. participants are intentionally selected who share a common characteristic of research interest [9]. Data from these multiple perspectives are then consolidated and used to convey the various perspectives among and between individuals within this particular group. Information is synthesized into categories and common themes to convey a general description of peoples "typical" experiences [4]. The study was conducted at the University Teaching Hospital out-patient Physiotherapy Department in Lusaka, Zambia.

\subsection{Study procedures}

Participants were purposefully sampled, where the researcher selected willing participants from the hospital because they were expected to be able to bring out the relevant information useful to the topic of study. Data collection was done by way of an interview guide and an audio recorder and then the data was later transcribed.

\subsection{Analysis}

Making use of the general inductive approach, thematic analysis was adopted for the study. The researcher generated information and then theories emerged from the data and obtained a conclusion. All the recorded interviews were transcribed and then the transcripts were read several times to identify some codes and the researcher coded by hand out of which themes emerged. Emerging themes were developed by studying the transcripts repeatedly and considering possible meanings and how these fitted with developing themes. The researcher finalized the names of the themes and wrote its description with a few quotations from the original text aid put across meaning to the reader.

\section{Results}

The participants were interviewed and guided by a semi-structured questionnaire where in some cases interviews involved probing of issues as they were mentioned by the participants. The researcher identified themes and other themes emerged from the views that the participants brought out. And then the findings were presented in a verbatim form as follows:

\subsection{Caregiver-Stroke survivor relationship and Support}

Interviews and discussions with the participants revealed that they were aware about the effect of caregiving on relationships. They were of the view that relationship with the stroke survivor can become closer, unchanged or distant during caregiving.

"I know that when one is caring for someone who has stroke, they usually become closer to them. They get to spend more time with the patient than before" (P01- Participant 1)

"Depending on the relationship that existed between the caregiver and the stroke survivor prior to stroke, the new relationship can be closer or further apart" (KI01-Key Informant 1)

"Being a mother, this role of caregiving has drawn me closer to my daughter...... when I wake up in the morning, I have to make sure that I prepare water for her to bath, prepare her breakfast...even when I knock off from work, I rush directly home to be with her" (P05-Participant 5)

They were also aware that during caregiving, there is usually moral, financial and

Brian C. Chiluba, Joseph Phiri. Tackling Disability of Speech due to Stroke: Perspectives from Stroke Caregivers of the University Teaching Hospital in Zambia. Indonesian Journal of Disability Studies (IJDS).2019: Vol. 6(2): PP 215-222. 
social support that is rendered to them. One participant narrated the following;

"Since the time my husband had a stroke, people such as friends and relatives have been supportive to us. They have physically visited us at home, helped financially and even prayed with us" (KI04-Key Informant 4)

3.2 Caregiver factors-such as initial expectations of the recovery of the stroke survivor, expression of feelings of depression, irritation etc

The interviews revealed that there were factors subjective to caregivers that posed as a challenge in effectively carrying out the role of caregiving. Caregivers revealed that it was not easy to see someone who was independent suddenly become helpless. They expressed feelings of depression, irritability, frustration, stress, anxiety, hopelessness etc.

"I usually get depressed to see my Auntie in her current state...she was physically okay but out of nowhere she has become helpless. She can no longer do the things she used to do by herself. When I look at her, I usually withdraw to a quiet place to just cry" (KI02-Key Informant 2)

Some caregivers revealed that it was hard for them to cope with the situation because they were uncertain about the recovery process.

"When my husband had the stroke, I thought he would get better in 3 months' time... right now I do not know when he will get back to the way he was" (P10-Participant 10)

Caregivers also reported stress and lack of social participation, usually as a consequence of managing and adjusting to the increased burden that occurred as a result of their competing demands and responsibilities, as well as the changed roles within and outside the home.

"Since the time my daughter had this stroke, I no longer visit friends, even family members...i get scared that she may have another stroke while I'm not around and end up dying" (KI05Key Informant 5)

Some caregivers stated that they had initial expectations when they assumed the role of caregiving and it was frustrating to see that most of their expectations were not fulfilled at the desired time.

"I thought my Uncle would be able to talk normally, use his hand and be able to walk normally after 2 months...it was frustrating to see that none of my expectations were fulfilled in 2 months" (P06-Participant 6)

\subsection{External factors-such as employment/business changes, financial costs} etc

During the discussions, it was noted that stressors outside the relational and support networks have also been found to impinge on caregiver's adjustment and wellbeing, such as employment/business changes.

"I was doing business of selling vegetables and other food staff but ever since my husband became sick, I had to stop so that I can attend to him fully" (KI03-Key Informant 3)

"I am a farmer...but since my son got sick, I left the farm and started staying with him so that I can care for him since he is not married" (P01Participant 1)

All the caregivers revealed that they got to spend more money during the time that their loved one had stroke and that it was not easy to find money especially for caregivers who were not working and those who stopped working or doing business in order to care for their loved one.

"It has been hard financially since my husband became sick......sometimes we go hungry at home just to reserve some money for bringing the patient to the hospital" (P10-Participant 10)

\subsection{Benefits of caregiving}

Amidst the pressures of caring for a stroke survivor, all the caregivers stated that they learnt something positive. Some of the benefits gained from the caregiving role includes;

"Taking care of my daughter has not been easy...I sometimes wonder where I draw the strength from. I sometimes feel like crying but I 
usually encourage myself that there is still hope. I cannot cry in front of my daughter because I am scared that she might get discouraged and stop putting effort in the treatment" (P05-Participant 5)

"Sometimes my husband gets so angry that he tries to hit me with a walking stick...i just ignore that because at the hospital I was told that when someone has had stroke, they start behaving like little children...i am just patient with him" (P10-Paricipant 10)

"The role of caregiving has helped us to rely completely on God...we have drawn closer to God than before and we pray regularly now" (P09-Participant 9)

\subsection{New Themes}

New themes emerged which were not reviewed in the literature and these are as follows;

Caregivers revealed that they were seriously affected by difficulties with the aphasic stroke survivor's memory, mood, motivation and personality (possibly as a result of poor executive functioning).

"Sometimes my husband loses the motivation...he does not agree to do some exercises at home and sometimes even refuses to be brought to the hospital "(KIO1-Key Informant 1)

"Sometimes my husband tries to hit me with a walking stick when I try to remind him about treatment sessions...even when I tell him to try walking the way he used to" (P09-Participant 9)

Caregivers reported that there are times when they feel dissatisfied with the hospital services they received.

"Sometimes physiotherapists especially female ones are rude... When we try to explain to them the patient's conditions when at home, they usually say it's because we do not carry out the home advise they tell us" (KI05-Key Informant 5)

"Most of us do not really understand what stroke is all about...we only see our loved ones unable to talk, use the hands and unable to walk... when we come to the hospital, we are always told to wait for our relatives from outside and no one who is skilled comes to even educate us about what our loved ones are suffering from" (P08-Participant 8).

Caregivers reported that caring for the stroke survivor is a great challenge especially that they also presented with speech problems. The stroke survivors were unable to utter nor write whatever they needed at any particular time, making it hard for the caregivers to know exactly what they needed. Caregivers also reported that due to failure of the stroke survivors to express themselves verbally, they were always frustrated and in rage making it hard for the caregivers to deliver quality care.

"Most of the times we do not know what the patient wants at a particular time...so we just include him in most of the activities at home e.g when it is time for family dinner, we also give him his share" (P07-Participant 7)

"Because I do not know what she wants all the time, I try to give her what I feel she should eat or wear but when she does not like it, she will just stand and go to the fridge or wardrobe to pick what she wants" (P05-Participant 5).

3.6 Root cause analysis diagram of the experiences of caregivers handling the aphasic stroke patients

The root cause diagram also known as the Ishikawa diagram is a visualization tool for categorizing the potential causes of a problem in order to identify its root causes. The following diagram shows what the caregivers handling aphasic stroke patients at the University Teaching Hospital went through since assumption of the caregiving role. 


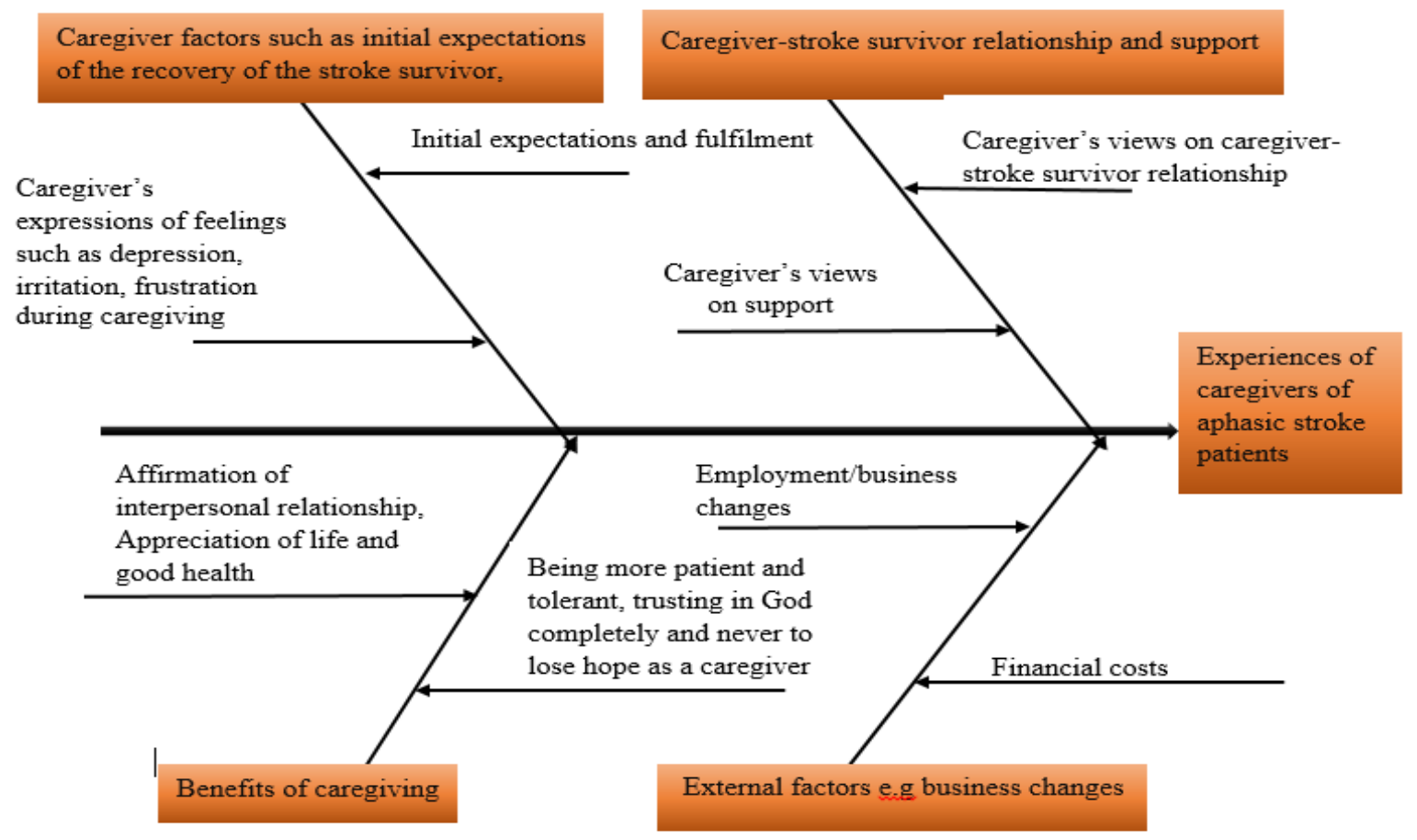

Figure 1. Root cause analysis diagram based on predetermined themes of experiences of caregivers of aphasic stroke patients.

\subsection{Discussion}

The results of the demographic characteristics of this study showed that more females are involved in caregiving compared to males, and most of these females are spouses. It also showed that there are more caregivers in the age range of 30-45 years compared to other age groups. This may mean that females fall victim to the consequences of caregiving and the survival of their loved ones largely depends on them. Females readily and easily assume the role of caregiving when their loved ones are in need of it. He further explains that this could possibly be attributed to their nature as emotional beings [10].

Caregivers stated that there was a change in the caregiver-stroke survivor relationship. The quality of this relationship was found to shift along the spectrum between distance/tension to closeness. The majority of the caregivers reported a development of a stronger relationship while others stated that their relationship became more distant, possibly due to poor communication. Caregivers with more stable relationships prior to the stroke usually care out of a sense of commitment and choice and their relationship become stronger and closer, whereas those with problematic premorbid relationships experience more distress, and usually feel obligated to be caregivers as there is no one else available to undertake the role [11].

Moral, financial and social support were some of the factors that influenced the caregivers in taking care of their loved ones. More specifically, caregivers became disappointed when family members and/or friends were not supportive enough, which sometimes led to relational tension. Caregivers asserted that informal support from family, friends and work colleagues also had a major impact on relationships, overall burden, ability to cope and emotional functioning [10].

Caregivers experienced depression, irritation, frustration, stress and anxiety while caring for their loved ones. These emotional patterns were usually explained by elements of grief and loss of a healthy partner/family member, personal independence and previous lifestyle (which included social relationships and specific roles once played). In addition, caregivers experienced internalized guilt that occurred if the stroke survivor was left alone or participated in their own separate leisure activities. The experience of juggling various demands and the negative changes in caregiver's mental and physical stability has a negative bearing in the efficacy of the role [9].

Uncertainty around the stroke survivor's recovery creates feelings of hopelessness and 
in particular, worried cognitions that something potentially detrimental would occur in the future, such as another stroke. This clearly shows that as the caregivers played their role, they in turn became vulnerable and succumbed to the emotional and physical break downs that caregiving brings along [12].

Caregivers' experience of stressors outside the relational and support networks impinged on their adjustment and wellbeing. These stressors included employment changes and the financial cost associated with caring for the stroke survivor. Caregivers employed at the time of their family member's stroke experienced reductions in workload while others stopped doing business in order to concentrate on the role of caregiving. This resulted in financial strain in providing basic needs at home and caring for the stroke survivor. This financial strain was greater, and their access to high quality stroke and rehabilitation services was also restricted. The role of caregiving encompasses various demands, among which is finances. He further explains that financial handicap and lack of resources during the time of caregiving places a great burden on caregivers which eventually deprives them of the zeal to render help effectively [13].

Qualitative research has predominantly focused on the negative impact of caregiving following a stroke [14], usually neglecting the benefits of the caregiving role. Nevertheless, the experience of caregiving is very complex and varies across individuals. Caregivers reported having benefited in the caregiving role in the following ways;

- They had affirmed interpersonal relationships with the aphasic stroke survivor and other people

- Increased their appreciation of life which involved a change in perspective and priorities

- Became more patient and tolerant with the stroke survivor

- Learnt to trust in God completely

- Learnt never to lose hope and being stronger as a caregiver

With particular regard to stroke caregivers, there is evidence of caregiver satisfaction with life and positive feelings about their role as a caregiver [15].
Caregivers reported that stroke survivors had difficulties with memory, mood, motivation and personality. This made it difficult for the caregivers to execute their role effectively. Due to lack of motivation, some stroke survivors refused to attend physiotherapy sessions at the hospital and did not comply with the home advice (exercises at home) given by the physiotherapists. For some stroke survivors who agreed to carry out home advice, they frequently forgot how to perform the exercises due to impaired memory. This frustrated the caregivers who at some point would contemplate on giving up on the caregiving role. This clearly shows that lack of compliance by the stroke survivor in the rehabilitative process has a negative effect on the overall well-being of the caregivers. It is possible that a stroke survivor's poor intrinsic motivation, confidence and outlook on their prognosis and recovery may sometimes cause poor compliance with rehabilitation regimes placing further burden and strain on the caregiver, who in turn, struggle to increase the patient's drive to recover [6].

Caregivers reported dissatisfaction with the quality of services, particularly therapistcaregiver communication. They stated that they did not know much about the condition of their loved ones because the physiotherapists were not willing enough to explain to them. Despite the dissatisfaction, they suggested that the best way of delivering stroke related information was in a face-to-face format. This is consistent with other studies which have found face-toface education and training for caregivers to be more efficacious than the provision of written materials [6].

Caregivers reported that due to failure of the stroke survivors to express themselves verbally, they were always frustrated and in rage, making it difficult for the caregivers to effectively care for them. Aphasia in stroke has proven to be a challenge and caregivers always report having difficulties in caring for such stroke survivors [16]. The stroke survivors are unable to utter nor write whatever they need at any particular time due to speech impairment and hemiplegia, making it difficult for the caregivers to know exactly what they needed. Patients with speech impairment usually exhibit their failure to communicate effectively through frustration and isolation $[17,18]$ and in order to care for such patients, caregivers need to be patient and

Brian C. Chiluba, Joseph Phiri. Tackling Disability of Speech due to Stroke: Perspectives from Stroke Caregivers of the University Teaching Hospital in Zambia. Indonesian Journal of Disability Studies (IJDS).2019: Vol. 6(2): PP 215-222. 
be able to closely observe their loved ones so that they may intuitively develop some skills in knowing what the patients want through gestures and other voluntary movements they may show [16]. Early speech therapy plays an integral role in the rehabilitative process of

All the participants reported that their quality of life had drastically reduced since they started their caregiving role. They neglected their physical, social, intellectual lives etc in order to focus wholly on the stroke survivors. Caregiving involves neglecting certain aspects of one's life such as personal and social attentions in order to fully concentrate on the stroke survivor $[6,19]$.

\subsection{Conclusion}

Caregivers go through a lot of things in caring for their loved ones and their experiences involve a complicated and sometimes unpredictable interplay of multiple processes. These processes occurred both within the individual such as the caregiver factors, as well as in the external environment such as interpersonal relationships with the stroke survivor and friends, the health care system, and financial stressors. The study has also shown that caregivers do not have prior knowledge or experience of caregiving before taking up the responsibility of caring for the stroke survivors. This results in them having to deal with completely new life situations unprepared for. Lack of preparedness caused the caregivers to go through what seemed to be insurmountable challenges. Furthermore, it has shown important themes that arise in the lives of stroke caregivers and this confirms the inadequacies of formal support services and resources, while at the same time highlighting what caregivers need and find beneficial. There is therefore need of caregiver education and social support so that they can deliver appropriate care to the stroke survivors. Amidst the negative experiences, the study has shown that caregivers also have positive experiences such as affirmation of interpersonal relationships with the aphasic stroke survivor, have increased their appreciation of life etc. Future research is needed to further test the proposed relationships between these factors through the use of relevant measures and in stroke survivors with aphasia [16].

specific clinical and psychological interventions.

\section{References}

National Stroke Foundation. (2015). Walk in our shoes: Stroke survivors and carers report on support after stroke. Melbourne, VIC: National Stroke Foundation.

Mackenzie A, Perry L, Lockhart E, Cottee M, Cloud G. (2014). Family caregivers of stroke survivors: needs, knowledge, satisfaction and competence in caring. Disability and Rehabilitation, 29(2), 111-121.

Ueda K, Teruo O, Hirota Y. (2015). Decreasing trend in incidence and mortality from stroke in Hisayama residents, Japan. Stroke, 12 (2): 154160.

Low J, Payne S, Roderick P. (2014). The impact of stroke on informal carers: A literature review. Social Science \& Medicine, 49, 711-725.

Hackett M, Yapa C, Parag V, Anderson C. (2013). The frequency of depression after stroke: a systematic review of observational studies. Stroke, 36, 1330-1340.

Christensen J, Hearn J, Bruno P. (2015). Projecting the number of patients with first ever strokes and patients newly handicapped by stroke in England and Wales. British Medical Journal, 298: 656-660.

Pinquart M, Sorensen S. (2006). Correlates of physical health of informal caregivers: a meta-analysis. The Journals of Gerontology, 62B, 126-137.

Visser-Meily A, Post M, Schepers V. (2014). Spouses' quality of life 1 year after stroke: Prediction at the start of clinical rehabilitation. Cerebrovascular diseases.

Dowswell G, Lawler J, Young J, Hearn J. (2010). A qualitative study of specialist nurse support for stroke patients and care-givers at home. Clinical Rehabilitation 11, 293-301.

Grant J, Bartolucci A, Elliott T. (2014). Sociodemographic, physical, and psychosocial characteristics of depressed and non-depressed

Cite this as:

Brian C. Chiluba, Joseph Phiri. Tackling Disability of Speech due to Stroke: Perspectives from Stroke Caregivers of the University Teaching Hospital in Zambia. Indonesian Journal of Disability Studies (IJDS).2019: Vol. 6(2): PP 215-222. 
family caregivers of stroke survivors. Brain Injury, 14(12), 1089-1100.

Pierce L, Steiner V, Hicks B, Holzaepfel A. (2007). Problems of new caregivers of persons with stroke. Rehabilitation Nursing, 31(4), 166172.

Scholte op Reimer M, Haan R, Rijinders P. (2016). The burden of caregiving in partners of long-term stroke survivors. Stroke, 29, 16051611.

Anderson M, Hearn S, Cloud G. (2016). Projecting the number of patients with first ever strokes and patients newly handicapped by stroke in England and Wales. British Medical Journal, 298: 656-660.

Parag D, Haan R. (2013). Shoulder pain after stroke: A review of the evidence base to inform the development of an integrated pathway". New York, USA: 111-115.

Han B, Haley W. (2010). Family caregiving for patients with stroke: Review and Analysis. Stroke; 30, 1478-1485.
Bugge C, Alexander H, Hagen S. (2011). Stroke Patient's Informal Caregivers. Patient, Caregiver and Service Factors that affect Caregiver strain. Stroke, 30, 1517-1523.

Alvarez J, Delgado P, Abilleira S, Molina C, Arenillas J, Ribo' M. (2016). Temporal profile of matrix metalloproteinases and their inhibitors after spontaneous intracerebral hemorrhage: relationship to clinical and radiological outcome. Stroke; 35:1316-22.

Chiluba BC. and Njapawu WG. (2019). Barriers of Persons with Physical Disability over Accessibility and Mobility to Public Buildings in Zambia. Indonesian Journal of Disability Studies (IJDS). Vol. 6(1): PP 53 - 63.

Chuni V. Chiluba BC. Mwango M. Nkandu EM. Shula H. (2018). An Exploration of the Knowledge of Informal Caregivers on Ageing Related Health Conditions at Matero and Chawama Old People's Homes, Zambia. International Journal of Neurologic Physical Therapy. Vol. 4, No. 1, pp. 1-6. doi: 10.11648/j.ijnpt.20180401.11

Brian C. Chiluba, Joseph Phiri. Tackling Disability of Speech due to Stroke: Perspectives from Stroke Caregivers of the University Teaching Hospital in Zambia. Indonesian Journal of Disability Studies (IJDS).2019: Vol. 6(2): PP 215-222. 